\title{
Genetic male infertility and mutation of CATSPER ion channels
}

\author{
Michael S Hildebrand ${ }^{1,2,9}$, Matthew R Avenarius ${ }^{3,9}$, Marc Fellous ${ }^{4,9}$, Yuzhou Zhang ${ }^{1,2}$, Nicole C Meyer ${ }^{1,2}$, \\ Jana Auer ${ }^{4,5}$, Catherine Serres ${ }^{4,5}$, Kimia Kahrizi ${ }^{6}$, Hossein Najmabadi ${ }^{6}$, Jacques S Beckmann ${ }^{7,9}$ \\ and Richard JH Smith ${ }^{\star, 1,2,8,9}$
}

A clinically significant proportion of couples experience difficulty in conceiving a child. In about half of these cases male infertility is the cause and often genetic factors are involved. Despite advances in clinical diagnostics $\sim 50 \%$ of male infertility cases remain idiopathic. Based on this, further analysis of infertile males is required to identify new genetic factors involved in male infertility. This review focuses on cation channel of sperm (CATSPER)-related male infertility. It is based on PubMed literature searches using the keywords 'CATSPER', 'male infertility', 'male contraception', 'immunocontraception' and 'pharmacologic contraception' (publication dates from January 1979 to December 2009). Previously, contiguous gene deletions including the CATSPER2 gene implicated the sperm-specific CATSPER channel in syndromic male infertility (SMI). Recently, we identified insertion mutations of the CATSPER1 gene in families with recessively inherited nonsyndromic male infertility (NSMI). The CATSPER channel therefore represents a novel human male fertility factor. In this review we summarize the genetic and clinical data showing the role of CATSPER mutation in human forms of NSMI and SMI. In addition, we discuss clinical management and therapeutic options for these patients. Finally, we describe how the CATSPER channel could be used as a target for development of a male contraceptive.

European Journal of Human Genetics (2010) 18, 1178-1184; doi:10.1038/ejhg.2010.108; published online 21 July 2010

Keywords: CatSper; male infertility; sperm motility; contraception

\section{INTRODUCTION}

Genetic male infertility

Human infertility. An essential step in the propagation of all vertebrate species is the union of a sperm and an oocyte to form a zygote. Before fertilization a series of highly regulated processes must occur that lead to embryonic development of the germ-line lineage and formation of the genital tracts and future gonads. Abnormalities associated with any of these processes can have severe consequences for the intended life plan of otherwise healthy couples. Although availability of assisted reproductive technologies (ARTs) has provided children for many infertile couples, it has also increased transmission of genetic defects. Despite improved understanding of these genetic defects and the molecular mechanisms they perturb, these advances have not been matched by progress in diagnosis and treatment of infertility.

The translation of basic research findings to improved clinical care for infertility in humans has been slow. Although in recent times our knowledge of complex reproductive processes including sex determination, gametogenesis, gamete function and fertilization has significantly increased, the major treatment options for infertile couples remain ART, surgical intervention and endocrine manipulation. ${ }^{1}$ In addition to these defects, premature menopause and the finite period of female reproductive capability have also contributed to infertility. And while many causes for infertility have been ascribed, particularly in the female, such as recurrent pregnancy loss, endometriosis, ovulatory defects, polycystic ovarian disease, fertilization failure and implantation defects, their underlying mechanisms remain poorly understood. ${ }^{1}$ Most often their diagnosis is descriptive or idiopathic, and the default treatment is ART.

Common genetic and environmental causes of male infertility. In approximately half of the $15 \%$ of couples who cannot conceive, the cause is ascribed to male infertility. ${ }^{2,3}$ The underlying pathogenetic mechanisms can reflect the effect of environmental toxins such as pesticides, glycol ethers and heavy metals; systemic disorders like hypothalamic-pituitary disease, testicular cancers and germ-cell aplasia; and genetic factors including aneuploidies and single-gene mutations (Table 1). ${ }^{4} \mathrm{Y}$ chromosome microdeletions cause infertility in $\sim 5 \%$ of patients, ${ }^{5}$ whereas mutations in the CFTR gene that lead to congenital bilateral absence of vas deferens and obstructive azoospermia (absence of sperm) account for $\sim 2 \%$ of infertile male patients. ${ }^{6}$

Of the aneuploidies associated with male infertility, the classic example is Klinefelter's syndrome., ${ }^{7,8}$ The presence of an extra $\mathrm{X}$ chromosome in these 47, XXY males leads to destruction of the

\footnotetext{
${ }^{1}$ Department of Internal Medicine, University of lowa, lowa City, IA, USA; ${ }^{2}$ Department of Otolaryngology, University of lowa, lowa City, IA, USA; ${ }^{3}$ Department of Human Genetics, University of Michigan School of Medicine, Ann Arbor, MI, USA; ${ }^{4}$ INSERM U567/CNRS UMR8104 Institut Cochin, Paris, France; ${ }^{5}$ Faculty of Medicine, University Paris Descartes, Paris, France; ${ }^{6}$ Genetics Research Center, University of Social Welfare and Rehabilitation Sciences, Tehran, Iran; ${ }^{7}$ Department of Medical Genetics, University of Lausanne and Centre Hospitalier Universitaire Vaudois, Lausanne, Switzerland; ${ }^{8}$ Interdepartmental PhD Program in Genetics, Department of Otolaryngology, University of Iowa, Iowa City, IA, USA

${ }^{*}$ Correspondence: Dr R Smith, Department of Internal Medicine, University of lowa, lowa City, IA 52242, USA. Tel: +1 3193563612 ; Fax: +1 3193564547 ;

E-mail: richard-smith@uiowa.edu

${ }^{9}$ These authors contributed equally to this work

Received 11 February 2010; revised 27 April 2010; accepted 4 June 2010; published online 21 July 2010
} 
seminiferous epithelium and azoospermia (absence of sperm). Single gene defects associated with azoospermia and asthenozoospermia (low sperm motility) include mutations in the A Kinase Anchoring Protein AKAP3 (OMIM 604689) and AKAP4 (OMIM 300185) (reviewed in Matzuk and Lamb ${ }^{1}$ ). In both humans and mice, deletions in these two genes cause sperm immotility secondary to dysplasia of the fibrous sheath surrounding the axoneme., ${ }^{9,10,1}$ Mutations in the dynein genes that encode proteins of the axonemal dynein cluster (DNAI1 (OMIM 604366), DNAH5 (OMIM 603335) and DNAH11 (OMIM 603339)) have been identified in persons with Kartagener's syndrome (OMIM 244400) and also cause asthenozoospermia. ${ }^{11,12}$

Genetic defects leading to male infertility often affect spermatogenesis or sperm function (reviewed in Matzuk and Lamb ${ }^{1}$ ). Many of these genes are required for normal sperm motility. The motility of mature mammalian spermatozoa is established upon their ejaculation and entry into the female reproductive tract. Once sperm enter the female reproductive tract, they become competent to fertilize the oocyte in a process termed 'capacitation' that involves three $\mathrm{Ca}^{2+}$-mediated events: initial activation, hyperactivation of motility and engagement of a protein kinase cascade. ${ }^{13}$ These events depend on changes in intracellular $\mathrm{Ca}^{2+}$, cAMP and $\mathrm{pH}$ levels (reviewed in Navarro et $\left.a^{14}\right)$. Genetic defects that impair any of these events lead to male infertility.

Table 1 Major genetic and environmental causes of male infertility

\begin{tabular}{ll}
\hline Genetic & Environmental \\
\hline $\begin{array}{l}\text { Obstruction of the ejaculatory ducts } \\
\text { (eg cystic fibrosis (CF), congenital bilateral }\end{array}$ & $\begin{array}{l}\text { Exposure to environmental toxins } \\
\text { (eg pesticides, glycol ethers, heavy } \\
\text { absence of the vas deferens (CBAVD)) }\end{array}$ \\
$\begin{array}{l}\text { Y-chromosome microdeletion } \\
\text { Endocrine abnormalities (eg congenital }\end{array}$ & agents, heat exposure) \\
adrenal hyperplasia, isolated follicle-stimu- & (eg mumps orchitis, epididymitis, \\
lating hormone (FSH) deficiency, & urethritis) \\
hyperprolactinemia) & Immunologic abnormalities \\
Hypothalamic-pituitary disease & (eg antisperm antibodies) \\
Vascular abnormalities (eg varicocele) & Trauma \\
Testicular tumors & \\
Germ-cell aplasia & \\
Sertoli-cell-only syndrome (SCOS) & \\
Aneuploidies (eg Klinefelter's syndrome & \\
(XXY)) & \\
Single-gene mutations (eg CATSPER1, & \\
AKAP3/4, DNAH1/5/11) &
\end{tabular}

\section{METHODS}

This review focuses on cation channel of sperm (CATSPER)-related male infertility. Semen analysis results, sperm motility and morphology characteristics, genetic diagnostics and family history data are reviewed and compared. The information on CATSPER function in sperm and potential development of male contraception is based on PubMed searches (http://www.ncbi.nlm.nih. gov/pubmed) using the keywords 'CATSPER', 'male infertility', 'male contraception', 'immunocontraception' and 'pharmacologic contraception' (publication dates from January 1979 to December 2009) and other relevant literature known to the authors. The literature was restricted to the articles in English and French.

\section{RESULTS AND DISCUSSION}

\section{CATSPER-related male infertility}

CATSPER mutations and male infertility. Mutations affecting the sperm-specific CATSPER1 (OMIM 606389) and CATSPER2 (OMIM 607249) alkalinization-activated calcium channels, two of four members of the CATSPER family, have been associated with male infertility in mice and humans (Table 2; Figure 1). Although mutation of CATSPER 1 has been associated with nonsyndromic male infertility (NSMI), ${ }^{15}$ loss of CATPSER2 and STRC in contiguous gene deletion syndromes leads to deafness-infertility syndrome (DIS) (OMIM 611102) (Table 2). ${ }^{16,17}$ In mice, genetic disruption of any one of the four sperm-specific CatSper channels (CatSper1/2/3/4) leads to male infertility by impairing sperm motility. ${ }^{18,19}$ To date, no mutations in CATSPER3 or CATSPER4 have been identified in human males with infertility.

The CATSPER proteins are a family of four sperm-specific alkalinization-activated cation channels (CATSPER-1, -2, -3, -4) that are highly conserved in humans and mice. ${ }^{20,21}$ CATSPER proteins consist of a single, six-transmembrane-spanning repeat with a P-loop between transmembrane domains S5 and S6 that shows homology to fourrepeat calcium $\left(\mathrm{Ca}_{\mathrm{v}}\right)$ channels. ${ }^{21}$ The mutant CATSPER1 proteins predicted for the NSMI families lack all six transmembrane domains and the P-loop. ${ }^{15}$ Thus, even if a truncated protein is made, it is predicted that CATSPER1 channel activity would be abolished in homozygous carriers of either of these mutations. Likewise, in DIS patients, part or all of the CATSPER2 gene is deleted, leading to predicted loss of the CATSPER2 protein.

The expression of CatSper1 is restricted to the plasma membrane of spermatozoa above the fibrous sheath in the principal piece of the mature sperm tail (Figure 1). ${ }^{21}$ CatSper proteins are also known to be expressed during the meiotic stages of spermatogenesis. ${ }^{22}$ Its functionality was confirmed by Carlson et $a l^{13}$ and Ren et $a l^{21}$ in experiments that showed the requirement of CatSper1 for $\mathrm{Ca}^{2+}$

Table 2 Human mutations reported in CATSPER 1/2

\begin{tabular}{|c|c|c|c|c|c|c|c|c|}
\hline Family & Mutation & Age of onset (infertility) & Pathology & Tissue expression & Population & Random controls & NMSI/DIS families & Reference \\
\hline \multicolumn{9}{|l|}{ CATSPER 1} \\
\hline Iranian L-1025 & p.Lys180LysfsX8 & Sexual maturity & NSMI & Sperm cells & Iranian & $0 / 576$ & $1 / 1$ & 15 \\
\hline Iranian L-968 & p.Arg180X (R180X) & Sexual maturity & NSMI & Sperm cells & Iranian & $0 / 576$ & $1 / 1$ & 15 \\
\hline \multicolumn{9}{|l|}{ CATSPER2 } \\
\hline French & Del (15)(q15.1-q15.3) & Sexual maturity & DIS & Sperm cells & French & Not reported & $1 / 1$ & 17 \\
\hline Iranian D_SM & Del (15)(q15.1-q15.3) & Sexual maturity & DIS & Sperm cells & Iranian & Not reported & $1 / 3$ & 16 \\
\hline Iranian L-705 & Del (15)(q15.1-q15.3) & Sexual maturity & DIS & Sperm cells & Iranian & Not reported & $1 / 3$ & 16 \\
\hline Iranian L-1014 & Del (15)(q15.1-q15.3) & Sexual maturity & DIS & Sperm cells & Iranian & Not reported & $1 / 3$ & 16 \\
\hline
\end{tabular}



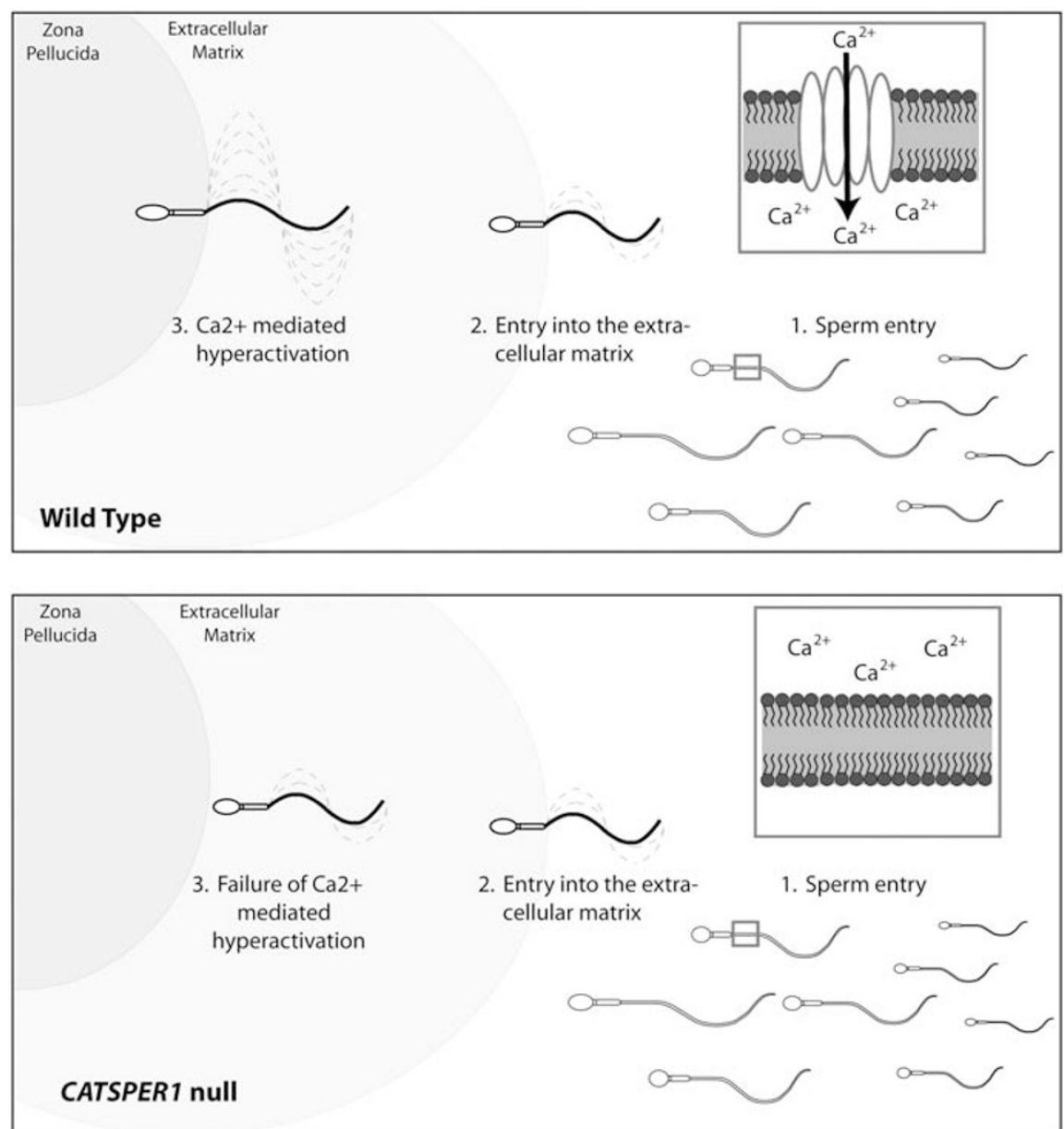

Figure 1 Sperm motility defects due to loss of CATSPER channel. CATSPER proteins are expressed in the principal piece of the sperm flagellum (box). Sperm deficient for CATSPER channels can move through the extracellular matrix (ECM) of the oocyte but cannot penetrate the zona pellucida due to failure to achieve $\mathrm{Ca}^{2+}$-dependent hyperactivated motility.

entry into the flagellum and for $\mathrm{Ca}^{2+}$-mediated hyperactivated sperm motility. Hyperactivated motility occurs at the site of fertilization to facilitate sperm penetration into the oocyte and is characterized by a high-amplitude, asymmetric waveform of the flagellum. Absence of CatSper1 impairs this phase of motility (Figure 1). ${ }^{13}$

Definitive evidence that CatSper1 functions as a alkalinizationactivated calcium channel to facilitate hyperactivated sperm motility came from whole-cell patch-clamp measurements of spermatozoa. ${ }^{23} \mathrm{~A}$ $\mathrm{Ca}^{2+}$ current $\left(I_{\text {CatSper }}\right)$ was recorded from the principal piece of the wild-type mouse sperm tail but not from CatSper-null sperm. $I_{\text {CatSper }}$ current was potentiated in response to intracellular alkalinization, increasing the concentration of intraflagellar calcium and thereby inducing hyperactivation. Although these were murine studies, the high degree of conservation of CatSperl between mouse and human $(55 \% \text { identity/72\% similarity })^{21}$ suggests a similar mechanism may be responsible for hyperactivated sperm motility in human males. While writing this review the first patch-clamp recording of a proton conductance by a voltage-gated channel in human spermatozoa was reported. ${ }^{24}$ This current induces an intracellular alkalinization by acid extrusion that together with CatSper channels contributes to hyperactivity of sperm.

Because mutation of CATSPER1 and CATSPER2 has now been associated with human male infertility, we provide here a brief summary of that published data ${ }^{16,17}$ and compare the clinical presentation of the human patients with CATSPER1- and CATSPER2related male infertility to the phenotype of CatSper mouse models. Although the role of CatSper in capacitation and fertilization has been well documented in the mouse, ${ }^{21-23}$ much less is known about the function of these channels in human sperm. Two studies have shown a correlation between CATSPER1/2/3 expression level and sperm motility in human patients, but these constitute the most comprehensive studies reported. ${ }^{25,26}$ This lack of knowledge not only reflects difficulty in identifying families with heritable forms of male infertility but also the inability to study reproductive processes within the human female reproductive tract. ${ }^{1}$ The relatively recent discovery of human families segregating male infertility due to disruption of the CATSPER1 or CATSPER2 genes has provided new insights that are worthy of discussion.

CATSPER1 and nonsyndromic male infertility. The CatSper1 channel was first identified for its role in sperm motility in $2001^{21}$ and its function in calcium transport was verified in 2006. ${ }^{23}$ However, involvement of this channel in human male fertility was only shown very recently in two consanguineous Iranian families. ${ }^{15}$ In both families insertion mutations were identified in exon 1 (c. 539-540insT and c. 948-949insATGGC) that lead to frameshift 
and premature stop codons. Severely truncated CATSPER1 protein lacking all transmembrane domains and the channel pore or complete absence of the protein due to nonsense-mediated decay is predicted in these patients. Routine analysis of semen from an affected male patient in each family segregating insertion mutations in CATSPER1 showed sperm defects and reduced fertility suggestive of asthenozoospermia (Table 3). The defects included nonmotile sperm or sperm motility below the normal threshold, low sperm count, increased abnormally structured spermatozoa and reduced semen volume. ${ }^{15}$ The $\mathrm{pH}$ of the semen of both individuals was within the normal range.

Although this rudimentary semen examination provided evidence of infertility, more rigorous investigation of sperm motility including measurement of progressive velocity, track speed and path velocity is needed to reveal the precise phenotype of the sperm in these patients. CatSper1-null mouse sperm show abnormalities in all of these measurable parameters. ${ }^{21}$ However, similar measurements could not be carried out from the sperm of affected males in these Iranian families due to technological limitations. In future studies, we could attempt to detect CatSper in the sperm of these patients using anti-CatSper antibodies developed to cross-react with the human proteins. ${ }^{19,21,22}$

CATSPER2 and syndromic male infertility. Syndromic male infertility (SMI) in association with CATSPER mutations was first described in a French family in which some individuals had a complex phenotype that included infertility, deafness and congenital dyserythropoietic anemia type 1 (CDA1) (Table 2). ${ }^{17}$ The CDA1 in affected members of this family was attributable to a homozygous p.N598S mutation in the $C D A I$ gene. The affected male patients were also homozygous for an $\sim 70 \mathrm{~kb}$ deletion of the proximal copy of a $106 \mathrm{~kb}$ tandem repeat on chromosome 15 (Del (15)(q15.1-q15.3)). The deleted region included four genes - the last two exons of CATSPER2, the entire coding sequence of $S T R C$ (stéréocilin protein) and CKMT1, and the first 24 exons of KIAA0377. The copies of these genes on the distal tandem repeat are pseudogenes. Similar (though not identical) deletions have also been reported in three unrelated Iranian families who segregate DIS (Table 2). ${ }^{16}$ The deletions in these families at $15 \mathrm{q} 15.3$ range from 90 to $100 \mathrm{~kb}$ and all include the complete loss of STRC and CATSPER2 at the genomic level. Mutation of STRC causes human nonsyndromic hearing loss at the DFNB16 locus, ${ }^{27}$ and CatSper ${ }^{-/-}$male mice are infertile, $^{22}$ implicating the disruption of both genes in the pathogenesis of DIS in these human families.
Routine semen analysis carried out on two of the three brothers in the French family revealed normal semen volume and $\mathrm{pH}$ but a number of sperm abnormalities suggestive of asthenozoospermia (Table 3). ${ }^{17}$ The defects included a low sperm count for one patient, reduced sperm motility with a percentage of progressive spermatozoa below the normal threshold (35 and 5\%), reduced sperm viability (49 and 30\%) and a high level of abnormal sperm morphology. The major sperm abnormalities noted in these patients were coiled and angulated flagella. ${ }^{17}$

An interpretable analysis of sperm motility using computer-aided sperm analysis technology was not possible given the low motility and the high proportion of abnormally structured spermatozoa. Although immunofluorescence revealed the specific localization of CATSPER2 to the principal piece of control human sperm flagella, ${ }^{17}$ as had been described previously in mice, ${ }^{22}$ no flagellar staining was observed in the male gametes of the two patients, suggesting absence of CATSPER2 protein expression (anti-CatSper2 antibodies kindly provided by Timothy Quill).

A very similar clinical presentation was observed in affected men in the Iranian families that included malformed sperm with short, coiled flagella and reduced sperm motility. ${ }^{16}$ For example, in one patient $>88 \%$ sperm were malformed (mainly thin heads, microacrosomes and irregular acrosomes) and $\sim 30 \%$ of sperm had short, coiled flagella. ${ }^{16}$ Following liquefaction $<5 \%$ of sperm had full swimming capacity. The malformed spermatozoa in patients with contiguous deletions, which include CATSPER2, suggests that in addition to a role in hyperactivated motility, CATSPER proteins are also important for the normal development of spermatozoa. ${ }^{17,22}$ Consistent with this CatSper2 is expressed in meiotic cells as well as spermatozoa. ${ }^{22}$

The hearing loss in DIS is prelingual and nonprogressive, and vestibular function is normal. In all reported affected men, the degree of hearing loss is moderate-to-severe across all frequencies $(0.25-8 \mathrm{kHz}) .{ }^{16,17}$ This auditory phenotype is comparable to that observed in persons with DFNB16. ${ }^{27,28}$

CatSper and infertility in mice. These clinical presentations are consistent with the phenotypes of CatSper-null mice, which have been described in detail previously. ${ }^{19,21,29}$ CatSper1 ${ }^{-1-}$ mouse sperm were sluggish, showed less directed movement and exhibited impaired track speed, path velocity and progressive velocity. ${ }^{21}$ This markedly reduced motility was shown to eliminate the ability of CatSper-null sperm to fertilize oocytes in vitro. ${ }^{21}$ Subsequent investigation showed

Table 3 Semen analysis of infertile patients with mutations reported in CATSPER 1/2

\begin{tabular}{|c|c|c|c|c|c|c|c|c|}
\hline Test & Normal range ${ }^{a}$ & $L-1025$ & $L-968$ & Patient 1 & Patient 2 & $D \_S M$ & $L 1014$ & L 1014 \\
\hline Ejaculate volume (ml) & $1.5-5$ & 1 & 0.4 & 2.8 & 4 & 4 & 2.2 & 1 \\
\hline $\mathrm{pH}$ & $>7.2$ & 8.0 & 7.5 & 7.2 & 7.4 & & t determir & \\
\hline Sperm concentration (million/ml) & $>20$ & 12 & 25 & 7.5 & 159 & 78 & 73 & 60 \\
\hline Normal morphology (\% normal) & $>30$ & 65 & 20 & 0 & 10 & 11 & 7 & 11 \\
\hline Viscosity (scale 0-4) & $<3$ & \multicolumn{2}{|c|}{ Not determined } & Normal & Normal & Normal & $3+$ & Normal \\
\hline
\end{tabular}

avalues from ASRM Practice Committee ${ }^{45}$ 
that the inability of both CatSper1 and CatSper2-null sperm to fertilize oocytes was directly attributable to the failure of hyperactivation due to an absence of depolarization-evoked $\mathrm{Ca}^{2+}$ entry. ${ }^{13}$ The lack of CatSper was specific for this motility defect as spermatogenesis and initial motility were normal in these mice. ${ }^{13}$ Targeted disruption of CatSper3 or CatSper 4 was associated with identical infertility in male mice. ${ }^{19}$ Taken together, these results suggest that the CATSPER 1 and CATSPER2 proteins also assemble to form a functional calcium channel in human sperm, which is essential for normal fertility, and possibly that the genes encoding for the additional CATSPER proteins are good candidates for additional human infertility genes.

\section{Clinical diagnosis and management of CATSPER-related male infertility}

Clinical diagnosis. The clinical evaluation of fertility in male patients is commonly limited to routine semen analysis to assess the ejaculate for abnormalities in sperm number, morphology and motility, as well as indicators of the function of the genital tract including semen volume and $\mathrm{pH}$ (Table 3 ). ${ }^{30}$ These measures are relatively rudimentary, and although semen analysis is effective for determination of azoospermia, changes in sperm morphology and motility can be missed. Despite the fact that a significant number of gene products are now associated with infertility in men (reviewed in Matzuk and $\mathrm{Lamb}^{1}$ ), many of these etiologies go undiagnosed in the absence of a more rigorous clinical examination that includes measurement of sperm motility parameters like path velocity, progressive velocity and track speed. These parameters are all markedly impaired in CatSper $1^{-/-}$ mouse sperm compared with wild-type sperm. ${ }^{21}$ If the appropriate technology is available, examination of these motility parameters, including determination of the percentage of hyperactivated cells, in human male patients with suspected CATSPER-related infertility is recommended.

For patients with suspected CATSPER2-related male infertility the diagnostic criteria described above for CATSPER-related NSMI should be used. However, as the contiguous gene deletion that includes CATSPER2 also includes STRC and mutation or deletion of STRC is associated with hearing loss, otological examination and audiological assessment should be completed. ${ }^{31}$ It is also possible that point mutations in CATSPER2 could be associated with infertility in the absence of hearing loss, although no such mutations have been reported.

Genotype-phenotype correlations. Because only a small number of mutations for CATSPER-related NSMI $(n=2)$ and DIS $(n=4)$ have been identified, knowledge of genotype-phenotype correlations for these diseases is limited. ${ }^{31}$ Nonsense mutations of CATSPER 1 are predicted to result in NSMI. ${ }^{15}$ It is not known whether less disruptive gene alterations such as missense mutations in CATSPER1 also lead to NSMI. In all known cases of DIS, contiguous gene deletions involving part or all of the CATSPER2 gene have been identified. ${ }^{16,17}$ It is not known whether point (nonsense or missense) mutations in CATSPER2 alone lead to NSMI.

Penetrance. The penetrance of NSMI caused by mutations in CATSPER1 is complete in male patients homozygous for a nonsense mutation (Table 2). ${ }^{15}$ Female patients homozygous for the mutation and all heterozygous individuals within a family are likely to have normal fertility. ${ }^{31}$ All male patients homozygous for CATSPER2 deletion show infertility as part of DIS. The penetrance for the hearing loss in male and female patients homozygous for the deletion of STRC in DIS is also $100 \%$, although onset and severity of hearing loss between individuals may vary. ${ }^{31}$
Genetic screening. For patients with suspected CATSPER-related male infertility molecular genetic testing should be conducted to establish the causative gene. Because mutations in a large number of genes cause male infertility (a partial list includes CATSPER1, AKAP3, AKAP4, DNAH1, DNAH5, DNAH11, SPATA16, PRM1, PRM2, SYCP1 and SYCP3), multiple genes can be considered for testing if such testing is available. ${ }^{31}$ Our CLIA-approved and Joint Commissionaccredited Molecular Otolaryngology Research Laboratories located at the University of Iowa Hospitals and Clinics currently offer genetic diagnostic tests for both CATSPER1- and CATSPER2-related infertility. $^{31}$

Clinical management and treatment options. There are no treatments available to reverse the morphological and/or motility defects observed in CATSPER-related asthenozoospermia. For infertile male patients, the only option to achieve conception is to bypass these motility defects using ARTs such as intracytoplasmic sperm injection (ICSI). ${ }^{16}$ In ICSI, a single ejaculated or nonejaculated (epididymal- or testicularderived) spermatozoon is injected directly through the oocyte membrane to achieve fertilization. ${ }^{32}$ Because the technique permits delivery of male chromosomes through the zona pellucida and oolemma into the ooplasm, sperm quality or motility issues are negated provided viable sperm are available from the male patient. ${ }^{33}$

Intracytoplasmic sperm injection represents an effective treatment for severe male factor infertility such as that observed in patients with CATSPER mutations and is now a routine procedure compatible with normal embryonic development. ${ }^{34}$ There are conflicting reports on the risk of congenital birth defects associated with ICSI. A relatively low $(4.2 \%)$ increased risk of congenital abnormalities for children conceived using ICSI has been reported in the largest multicenter study. ${ }^{35}$ Whether this risk relates to the ICSI procedure directly or inherent properties of the sperm or egg is unclear. However, this type of information should be provided to couples considering this procedure. ICSI has been successfully used in male patients with DIS. ${ }^{16}$ Its use in male patients with CATSPER1-related NSMI has not been reported.

Genetic counseling. Because CATSPER-related NSMI and DIS are inherited in an autosomal recessive manner, individuals diagnosed with CATSPER-related NSMI or DIS typically do not have an infertile or deaf parent. ${ }^{31}$ Despite this the family history may appear to be negative because of failure to recognize the disorder in family members. A proband with NSMI or DIS may have infertility and/or deafness as a result of a new gene mutation. The proportion of cases caused by de novo mutations is unknown. If the infertility- or deafness-causing mutation found in the proband cannot be detected in the DNA of either parent, two possible explanations are germ-line mosaicism in a parent or a de novo mutation in the proband. ${ }^{31}$ Although no instances of germ-line mosaicism have been reported, it remains a possibility.

The risk to the sibs of a proband being infertile and/or deaf depends on the use of ICSI as pregnancies have been achieved from males with DIS using ICSI. ${ }^{16}$ Each child with both parents carriers of a CATSPER mutation has a $25 \%$ chance of inheriting two copies of the diseasecausing mutation and a $50 \%$ chance of being a carrier. ${ }^{31}$ The risk to other family members depends on the status of the parents of the proband. If a father is found to be infertile or deaf, his male siblings may be at risk.

The optimal time for determination of genetic risk is before pregnancy. It is appropriate to offer genetic counseling (including discussion of potential risks to offspring and reproductive options) to 
young adults who are infertile or deaf. ${ }^{31}$ Pregnancies conceived by men with infertility due to CATSPER mutations through ART are at risk for CATSPER-related NSMI and DIS. Because both NSMI and DIS are recessive, recurrence chances can be predicted by screening the mother. Differences in perspective may exist among medical professionals and within families regarding the use of prenatal testing, particularly if the testing is being considered for the purpose of pregnancy termination rather than early diagnosis. ${ }^{31}$ Although most centers would consider decisions about prenatal testing to be the choice of the parents, careful discussion of these issues is appropriate. Preimplantation genetic diagnosis may soon be available for pregnancies conceived by male patients with infertility due to CATSPER mutations through ART and at risk of resulting in a male progeny with CATSPER mutations.

\section{CATSPER channels as a potential target for male contraception}

Male contraception. There is an urgent global need for a safe and effective male contraceptive. No pharmacological method is available for men despite the fact that effective female oral contraceptives have been available for more than 40 years. Although widespread use of female contraceptives, condoms and surgical contraception has reduced global population growth, the population continues to increase rapidly and is predicted to reach 8 billion by $2020 .{ }^{36}$ Millions of unintended pregnancies occur annually as a result of the lack of satisfactory contraceptive methods and in more than half of these cases the pregnancy is terminated by elective abortion. Complications during unintended pregnancies led to the death of $>700000$ women worldwide between 1995 and 2000. ${ }^{37}$ Most importantly for the potential implementation of a male contraceptive, studies have shown that men would be willing to use one if it were available. ${ }^{38}$

CATSPER immunocontraception. Several properties of the CATSPER channel make it an attractive target for development of a male contraceptive. ${ }^{14,39}$ The essential role of the channel protein in male fertility suggests an antibody or drug that blocks its activity would prevent fertilization. In addition, CATSPER expression is restricted to spermatogenic cells and mature sperm, eliminating any off-target effects in other parts of the body. ${ }^{21}$ Following ejaculation the CATSPER channel proteins remain accessible, suggesting a contraceptive agent delivered orally could reach the channels on the sperm surface. And, as CATSPER channels only function in mature sperm, reversible action of such an antibody or drug could be achieved if it is administered only while an individual is sexually active.

Immunocontraception targeted at the CATSPER channel is a potentially safe and viable method of male contraception. The idea of immunocontraception is not new and immunocontraceptive vaccines have already entered clinical trials (for review see Aitken ${ }^{40}$ ). A significant number of otherwise healthy infertile men and women have naturally occurring antisperm antibodies, showing the feasibility of this approach. ${ }^{41}$ In addition, immunocontraception would be easy to administer through existing disease vaccination programs, is reversible and long-acting. ${ }^{26}$

Although an immunocontraceptive for male infertility is currently unavailable in the clinic, a recent study has provided in vitro evidence that such an approach could work. On the basis of evidence that some autoantibodies block ion channels and cause autoimmune disease, ${ }^{42}$ Li et al ${ }^{26}$ tested the ability of anti-CatSper1 IgG (H-300) to inhibit CatSper activity in sperm. After showing the specificity of $\mathrm{H}-300$ against human and mouse CatSper protein, $50 \mu \mathrm{g} / \mathrm{ml}$ antibody was incubated with human and mouse sperm in vitro. The H-300 antibody binds to amino acids $481-780$ of the human CATSPER1 protein that encodes five of the six transmembrane domains and the pore region. Human and mouse CATSPER 1 proteins share $>80 \%$ identity and similarity for this region. These experiments revealed a decline in sperm progressive motility after 1,2 and $4 \mathrm{~h}$ of treatment with the antibody. In addition, a significant reduction in hyperactive motility was also observed in the treated sperm after $5 \mathrm{~h}$. To determine the effect of this inhibition on fertility, mouse sperm were treated with $20 \mu \mathrm{g} / \mathrm{ml} \mathrm{H}-300$ before insemination and a $72 \%$ reduction in the in vitro fertilization rate compared with untreated control sperm was observed. ${ }^{26}$

Taken together these results show the immunocontraceptive potential of CatSper1. The H-300 antibody is specific and its inhibitory effects on human sperm generate a similar motility phenotype to that reported for CatSper1-null mouse sperm. ${ }^{21}$ Incubation with the antibody significantly reduced the ability of mouse sperm to fertilize oocytes. Nonetheless, further testing of the safety and efficacy of $\mathrm{H}-300$ application in vivo is required to establish proof-of-principle CatSper-targeted immunocontraception.

CATSPER pharmacologic contraception. An alternative to immunocontraception is pharmacologic inhibition of CatSper channel activity. Some ion channels are attractive targets for male contraception based on their critical roles in sperm function and restricted expression to sperm (for review see Zhang and Gopalakrishnan ${ }^{43}$ ). The rationale for targeting such channel proteins is based on studies showing inadvertent modulation of these proteins can lead to reversible inhibition of fertility. One case reported is the $\mathrm{Ca}^{2+}$ channel blocker nifedipine that is prescribed for treatment of migraine and high blood pressure. During nifedipine treatment the male patient is infertile however fertility returns 3 months after administration ceases. ${ }^{44}$

A selective blocker of CatSper channel activity has recently been identified that could function as a pharmacologic male contraceptive. ${ }^{39}$ In a screen of proprietary compound libraries, Carlson and colleagues identified HC-056456 as a selective and reversible blocker of CatSper channel activity. By measuring channel currents in sperm, the authors showed that $3-10 \mu \mathrm{M}$ HC-056456 could inhibit alkaline depolarization-induced CatSper $\mathrm{Ca}^{2+}$ channel activity. The lack of effect on $\mathrm{HCO}_{3}{ }^{-}$-evoked, cAMP-mediated activation that leads to increased flagella beat frequency showed the specificity of HC-056456 as this activation is $\mathrm{Ca}^{2+}$ dependent but CatSper independent. ${ }^{39}$ Application of $20 \mu \mathrm{m}$ HC- 056456 blocked at least $50 \%$ of the observed CatSper current and strongly inhibited hyperactivated motility of the sperm in capacitating incubations as indicated by their reduced asymmetry. ${ }^{39}$ Importantly, the inhibitory action of the drug was reversible as sperm were able to recover waveform asymmetry $\sim 60 \mathrm{~s}$ after removal of HC-056456. Thus, the action of HC-056456 induces a selective late stage block on fertility and creates a phenocopy of CATSPER-null sperm.

A caveat of this approach is that HC-056456 is an imperfect selective blocker of CatSper channel activity. Investigation of the other major channel of sperm, Ksper, revealed that $50 \mu \mathrm{M} \mathrm{HC}$ 056456 partially blocked its potassium current. ${ }^{39}$ This reduced specificity has the potential to produce unwanted off-target effects in sperm and it will be necessary to engineer or identify more specific CatSper blockers similar to HC-056456 for use as pharmacologic male contraceptives. Nonetheless, this drug represents an exciting new development in the contraceptive field.

\section{CONCLUSION}

In cases of recessively inherited male infertility and DIS, mutations in the CATSPER channel genes should be considered a potential cause. CATSPER mutations may also be associated with sporadic cases of 
male infertility although there is no data on this in the literature. Because CATSPER mutations have only been identified in a handful of families, the overall contribution of this gene to male infertility is not established. Identification of families segregating male infertility is difficult but additional studies of large cohorts of sporadic infertile men should elucidate this contribution. The diagnosis of CATSPERrelated infertility is important because it provides patients with prognostic information and treatment options such as ICSI.

\section{CONFLICT OF INTEREST}

The authors declare no conflict of interest.

\section{ACKNOWLEDGEMENTS}

R Smith is the Sterba Hearing Research Professor, University of Iowa College of Medicine, supported financially by NIH grants RO1 DC03544, RO1 DC02842 and RO1 DK074409. M Hildebrand was supported by an Australian National Health and Medical Research (NHMRC) Postdoctoral Training Fellowship.

\section{AUTHOR CONTRIBUTIONS}

MSH, MRA and MF designed the review; YZ, NCM, JA, CS, KK and HN compiled and analyzed clinical data; and MSH, MRA, MF, JSB and RJHS wrote the article.

1 Matzuk MM, Lamb DJ: The biology of infertility: research advances and clinical challenges. Nat Med 2008; 14: 1197-1213.

2 Templeton A, Fraser C, Thompson B: The epidemiology of infertility in Aberdeen. BMJ 1990; 301: 148-152.

3 Mosher W, Pratt W: Fecundity and Infertility in the United States, 1965-1988. Advanced Data from Vital and Health Statistics. Hyattsville: National Center for Health Statistics, 1990.

4 Skakkebaek NE, Giwercman A, de Kretser D: Pathogenesis and management of male infertility. Lancet 1994; 343: 1473-1479.

5 Pryor JL, Kent-First M, Muallem A et al: Microdeletions in the $Y$ chromosome of infertile men. N Engl J Med 1997; 336: 534-539.

6 Shah K, Sivapalan G, Gibbons N, Tempest H, Griffin DK: The genetic basis of infertility. Reproduction 2003; 126: 13-25.

7 Arce B, Padron S: Spermatogenesis in Klinefelter's syndrome. Reproduction 1980; 4 : 177-184.

8 Grabski J, Pusch H, Schirren C et al: [Clinical, endocrinological, histological and chromosomal investigations on Klinefelter's syndromel. Andrologia 1979; 11: 182-196.

9 Turner RM, Musse MP, Mandal A et al: Molecular genetic analysis of two human sperm fibrous sheath proteins, AKAP4 and AKAP3, in men with dysplasia of the fibrous sheath. J Androl 2001; 22: 302-315.

10 Chemes HE, Brugo S, Zanchetti F, Carrere C, Lavieri JC: Dysplasia of the fibrous sheath: an ultrastructural defect of human spermatozoa associated with sperm immotility and primary sterility. Fertil Steril 1987; 48: 664-669.

11 Zuccarello D, Ferlin A, Cazzadore $C$ et al: Mutations in dynein genes in patients affected by isolated non-syndromic asthenozoospermia. Hum Reprod 2008; 23: 1957-1962.

12 Schwabe GC, Hoffmann K, Loges NT et al: Primary ciliary dyskinesia associated with normal axoneme ultrastructure is caused by DNAH11 mutations. Hum Mutat 2008; 29: 289-298.

13 Carlson AE, Westenbroek RE, Quill T et al: CatSper1 required for evoked Ca2+ entry and control of flagellar function in sperm. Proc Natl Acad Sci USA 2003; 100: $14864-14868$.

14 Navarro B, Kirichok Y, Chung JJ, Clapham DE: Ion channels that control fertility in mammalian spermatozoa. Int J Dev Biol 2008; 52: 607-613.

15 Avenarius MR, Hildebrand MS, Zhang $Y$ et al: Human male infertility caused by mutations in the CATSPER1 channel protein. Am J Hum Genet 2009; 84: 505-510.

16 Zhang Y, Malekpour M, Al-Madani N et al: Sensorineural deafness and male infertility: a contiguous gene deletion syndrome. J Med Genet 2007; 44: 233-240.

17 Avidan N, Tamary H, Dgany $\mathrm{O}$ et al: CATSPER2, a human autosomal nonsyndromic male infertility gene. Eur J Hum Genet 2003; 11: 497-502.
18 Jin J, Jin N, Zheng $\mathrm{H}$ et al: Catsper3 and Catsper4 are essential for sperm hyperactivated motility and male fertility in the mouse. Biol Reprod 2007; 77: 37-44.

19 Qi H, Moran MM, Navarro B et al: All four CatSper ion channel proteins are required for male fertility and sperm cell hyperactivated motility. Proc Natl Acad Sci USA 2007; 104: 1219-1223.

20 Lobley A, Pierron V, Reynolds L, Allen L, Michalovich D: Identification of human and mouse CatSper3 and CatSper4 genes: characterisation of a common interaction domain and evidence for expression in testis. Reprod Biol Endocrinol 2003; 1: 53.

21 Ren D, Navarro B, Perez G et al: A sperm ion channel required for sperm motility and male fertility. Nature 2001; 413: 603-609.

22 Quill TA, Ren D, Clapham DE, Garbers DL: A voltage-gated ion channel expressed specifically in spermatozoa. Proc Natl Acad Sci USA 2001; 98: 12527-12531.

23 Kirichok Y, Navarro B, Clapham DE: Whole-cell patch-clamp measurements of spermatozoa reveal an alkaline-activated $\mathrm{Ca}^{2+}$ channel. Nature 2006; 439: 737-740.

24 Lishko PV, Botchkina IL, Fedorenko A, Kirichok Y: Acid extrusion from human spermatozoa is mediated by flagellar voltage-gated proton channel. Cell 2010; 140: 327-337.

25 Nikpoor P, Mowla SJ, Movahedin M, Ziaee SA, Tiraihi T: CatSper gene expression in postnatal development of mouse testis and in subfertile men with deficient sperm motility. Hum Reprod 2004; 19: 124-128.

26 Li HG, Ding XF, Liao AH, Kong XB, Xiong CL: Expression of CatSper family transcripts in the mouse testis during post-natal development and human ejaculated spermatozoa: relationship to sperm motility. Mol Hum Reprod 2007; 13: 299-306.

27 Verpy E, Masmoudi S, Zwaenepoel I et al: Mutations in a new gene encoding a protein of the hair bundle cause non-syndromic deafness at the DFNB16 locus. Nat Genet 2001; 29: 345-349.

28 Villamar M, del Castillo I, Valle N, Romero L, Moreno F: Deafness locus DFNB16 is located on chromosome $15 q 13-q 21$ within a $5-\mathrm{cM}$ interval flanked by markers D15S994 and D15S132. Am J Hum Genet 1999; 64: 1238-1241.

29 Carlson AE, Quill TA, Westenbroek RE, Schuh SM, Hille B, Babcock DF: Identical phenotypes of CatSper1 and CatSper2 null sperm. J Biol Chem 2005; 280: 32238-32244.

30 WHO: World Health Organization Laboratory Manual for the Examination of Human Semen and Sperm-cervical Mucus Interaction. Cambridge: Cambridge University Press, 1999.

31 Hildebrand MS, Avenarius MR, Smith RJ: CATSPER-Related Male Infertility. Seattle: University of Washington, 2009.

32 Bonduelle M, Camus M, De Vos A et al: Seven years of intracytoplasmic sperm injection and follow-up of 1987 subsequent children. Hum Reprod 1999; 14: 243-264.

33 PCoASfRMPCoSfAR Technology: Genetic considerations related to intracytoplasmic sperm injection (ICSI). Fertil Steril 2008; 90: S182-S184.

34 Palermo GD, Colombero LT, Schattman GL, Davis OK, Rosenwaks Z: Evolution of pregnancies and initial follow-up of newborns delivered after intracytoplasmic sperm injection. JAMA 1996; 276: 1893-1897.

35 Bonduelle M, Wennerholm UB, Loft A et al: A multi-centre cohort study of the physical health of 5-year-old children conceived after intracytoplasmic sperm injection, in vitro fertilization and natural conception. Hum Reprod 2005; 20: 413-419.

36 Nieschlag E, Henke A: Hopes for male contraception. Lancet 2005; 365: 554-556.

37 Nass S, Strauss J: New Frontiers in Contraceptive Research: A Blueprint for Action. Washington, DC: Institute of Medicine National Academy Press, 2004.

38 Martin CW, Anderson RA, Cheng $L$ et al: Potential impact of hormonal male contraception: cross-cultural implications for development of novel preparations. Hum Reprod 2000; 15: 637-645.

39 Carlson AE, Burnett LA, del Camino $\mathrm{D}$ et al: Pharmacological targeting of native CatSper channels reveals a required role in maintenance of sperm hyperactivation. PLoS One 2009; 4: e6844.

40 Aitken RJ: Immunocontraceptive vaccines for human use. J Reprod Immunol 2002 ; 57: 273-287

41 Bronson R, Cooper G, Rosenfeld D: Sperm antibodies and infertility. Fertil Steril 1982; 37: 449-451.

$42 \mathrm{Li} \mathrm{H}$, Ding X, Guan H, Xiong C: Inhibition of human sperm function and mouse fertilization in vitro by an antibody against cation channel of sperm 1: the contraceptive potential of its transmembrane domains and pore region. Fertil Steril 2009; 92: 1141-1146.

43 Lang B, Vincent A: Autoantibodies to ion channels at the neuromuscular junction. Autoimmun Rev 2003; 2: 94-100.

44 Zhang D, Gopalakrishnan M: Sperm ion channels: molecular targets for the next generation of contraceptive medicines? J Androl 2005; 26: 643-653.

45 Hershlag A, Cooper GW, Benoff S: Pregnancy following discontinuation of a calcium channel blocker in the male partner. Hum Reprod 1995; 10: 599-606.

46 ARSM Practice Committee: Report on optimal evaluation of the infertile male. Fertil Steril 2006; 86: S202-S209. 